http://dx.doi.org/10.32929/2446-8355.2019v28n1p65-81

\title{
FOLHA ÍNDICE REPRESENTATIVA DO TEOR DE POTÁSSIO EM TOMATEIRO
}

Luiz Henrique Campos de Almeida ${ }^{1 *}$, Paula Pinheiro Sanches ${ }^{1}$, Mônica Mariana Jorge Fratoni $^{1}$, Eli Carlos de Oliveira ${ }^{1}$, André Prechlak Barbosa ${ }^{1}$, Greg Richardson ${ }^{2}$, Jaimie West ${ }^{2}$, Claudemir Zucareli ${ }^{3}$, Gustavo Adolfo de Freitas Fregonezi ${ }^{4}$.

\author{
1Pós-graduando, agronomia, Universidade Estadual de Londrina, Londrina-Paraná. Brasil. \\ *caluizhenrique@msn.com. \\ ${ }^{2}$ Pós-graduando, Soil science, University Of Wisconsin, Madison-Wisconsin. U.S.A. \\ ${ }^{3}$ Professor, doutor, Fitotecnia, Universidade Estadual de Londrina, Londrina-Paraná. Brasil. \\ ${ }^{4}$ Professor, doutor, Nutrição de plantas, UNIFIL, Londrina-Paraná. Brasil.
}

Recebido: 02/06/2017; Aceito: 14/03/2019

RESUMO: O potássio $(\mathrm{K})$ é um dos nutrientes mais exigidos pelas plantas, sendo responsável pela abertura estomática, translocação de açúcares, síntese proteica e ativação enzimática. Análises do teor disponibilizado para as plantas e sua relação com o que esta presente no tecido vegetal, através de folhas que melhor representam a planta nutricionalmente são alternativas para manejo correto de adubação. Porém, o estudo da folha ideal para este tipo de análise ainda é incipiente para o tomateiro. O objetivo do trabalho foi avaliar a folha que melhor representa o teor nutricional da planta visando produtividade. Foi avaliada a influência do aumento nas doses de $\mathrm{K}$ sobre a produção e teor de nutrientes nas folhas acima de cada cacho, até o terceiro cacho. Para o estudo foram utilizados dois experimentos com tomate fertirrigado com cinco doses de K, cultivado em casa de vegetação. Os valores foram submetidos à análise de variância e as médias à regressão polinomial até segundo grau e também à correlação. Os resultados mostraram que o aumento das doses de potássio influiu significativamente na produtividade, e nos teores de Potássio (K), Enxofre (S) e Nitrogenio $(\mathrm{N})$. Cada cultivar apresentou uma folha índice mais representativa visando a produtividade.

Palavras-chave: Tecido vegetal. Teor nutricional. Digestão nítrico-perclórica. Solanum esculentum Mill.

\section{NUTRITIONAL INDEX LEAF MORE REPRESENTATIVE IN TOMATO}

ABSTRACT: Potassium $(\mathrm{K})$ is one of the nutrients most required by plants, being responsible for stomatal opening, translocation of sugars, protein synthesis and enzymatic activation. Analysis of the content available for the plants and its relation with what is present in the plant tissue, through leaves that better represent the plant nutritionally are alternatives for correct management of fertilization. However, the study of the ideal leaf for this type of analysis is still incipient for the tomato. The objective of this work was to evaluate the leaf that best represents the nutritional content of the plant aiming at productivity. The influence of the increase in $\mathrm{K}$ rates on yield and nutrient content in leaves above each cluster was evaluated until the third bunch. For the study, two experiments were carried out with tomatoes 
fertiirrigado with five doses of $\mathrm{K}$, grown in greenhouse. The values were submitted to analysis of variance and the means to the polynomial regression until second degree and also to the correlation. The results showed that the increase of the potassium doses had a significant influence on yield, $\mathrm{K}, \mathrm{S}$ and $\mathrm{N}$ contents. Each cultivar presented a more representative index sheet for productivity.

Key words: Plant tissue. Nutritional content. Nitric-perchloric digestion. Solanum esculentum Mill.

\section{INTRODUÇÃO}

O tomate (Solanum esculentum Mill) é originário da América do Sul e uma das hortaliças mais consumidas no mundo, sendo que o Brasil está entre os dez maiores produtores mundiais (FAO, 2016; KAMALULDEENA et al., 2014).

Seu cultivo em casas de vegetação no período de entressafra pode propiciar produtividades superiores a $100 \mathrm{t} \mathrm{ha}^{-1}$ (ALMEIDA et al., 2016). Esta tecnologia de cultivo advém da necessidade de fornecer ao consumidor produtos in natura de boa qualidade durante todo o ano (FERNANDES et al., 2002).

Conforme Valérie et al. (2010), o intenso manejo do solo em casas de vegetação, acarreta a salinização do solo, em razão da fertilização em demasia e também a proliferação de fitopatógenos. Desta forma, o cultivo via fertirrigação em vasos contendo areia como substrato é uma opção para minimizar os danos, pois os nutrientes ficam restritos e, além disso, evita o contato da planta com o solo contaminado.

A fertirrigação consiste em adubar e irrigar ao mesmo tempo, sendo uma técnica consolidada, com vantagens, como: aplicação de nutrientes de acordo com as necessidades das plantas; aumento da eficiência dos fertilizantes; subdividir a aplicação ao longo do desenvolvimento da cultura; economia de água (BRAGA, 2010).

A desvantagem do método é o preço inicial da infraestrutura e a necessidade de mão de obra capacitada (VILLAS BOAS; SOUZA, 2008) para o manejo das soluções a fim de que não afete o potencial osmótico, prejudicando o fluxo de absorção de água pela planta (ANDRIOLO et al., 2005) e evitando problemas causados pela toxidez de alguns nutrientes (AYRES; WESTCOT, 1991).

Para aumentar a produtividade do tomate pela técnica da fertirrigação, todos nutrientes têm papel fundamental. Entretanto, o potássio (K) tem uma atribuição diferenciada, visto que é o mais exigido a partir do período reprodutivo e desempenha diversas funções nas células das plantas, atuando como co-fator enzimático, na síntese e estabilidade das proteínas e carboidratos (FAQUIN, 2001; TAIZ; ZEIGER, 2004).

Além disso, influencia a síntese de carotenoides, proporcionando cor vermelha ao fruto e também participa da síntese e transporte de açúcares (MARSHNER, 1995).

Assim, o conhecimento sobre o conteúdo nutricional das plantas é importante para avaliar a capacidade de remoção de nutrientes de cada cultura, e a quantidade que deve ser 
fornecida em cada etapa do cultivo para garantir altas produtividades (ALMEIDA et al., 2015)

O acompanhamento do teor de nutrientes e principalmente do K no cultivo fertirrigado é de suma importância para a reposição nutricional de acordo com as necessidades da cultura durante seu ciclo. Este se dá principalmente pela coleta e avaliação nutricional da folha índice do tomateiro, que é a que melhor representa a planta nutricionalmente.

Almeida et al. (2016) utilizaram a folha localizada logo acima do segundo cacho, já Ribeiro et al. (1999) assumem maior folha superior próxima do $3^{\circ}$ cacho como sendo a folha índice.

Diante das divergências na literatura sobre qual a melhor folha a ser utilizada para acompanhar o status nutricional do tomateiro o presente trabalho teve por objetivo avaliar a influência do aumento nas doses de $\mathrm{K}$ sobre a produção, absorção de nutrientes e teor nutricional de folhas de tomateiro afim de determinar a folha que melhor representa a planta nutricionalmente.

\section{MATERIAL E MÉTODOS}

Foram realizados dois experimentos, conduzidos em casa de vegetação, tipo arco, com 6 metros de largura, 30 metros de comprimento e cobertura de polietileno transparente, no Departamento de Agronomia da Universidade Estadual de Londrina. Amostras analisadas no Laboratório de Solos, em Londrina, PR, (latitude $23^{\circ} 23^{\prime} \mathrm{S}$, longitude $51^{\circ} 10^{\prime} \mathrm{W}$, com altitude de 580 metros (ALMEIDA et al., 2016).

Estes experimentos consistiram em testes de aumento de dose de $\mathrm{K}$ com duas cultivares de tomateiro fertirrigados cultivados em vasos com areia. Foram instalados em delineamento de blocos ao acaso, com cinco tratamentos e 10 repetições, totalizando 50 vasos lado a lado e $60 \mathrm{~cm}$ entre linhas, com bordaduras ao redor e fertirrigados por gotejamento.

As plantas foram cultivadas em vasos plásticos com capacidade para $9 \mathrm{~L}$ (23,5 cm x 26 $\mathrm{cm} \times 19,5 \mathrm{~cm}$ ), utilizando areia de granulometria grossa como substrato. Os resultados da análise da areia obtidos foram: $\mathrm{H}^{+}+\mathrm{Al}^{+3}=1,89 \mathrm{cmol}_{\mathrm{c}} \mathrm{dm}^{-3}$; matéria orgânica $=0 \mathrm{~g} \mathrm{dm}^{-3} ; \mathrm{K}^{+}=$ $0 \mathrm{cmol}_{\mathrm{c}} \mathrm{dm}^{-3} ; \mathrm{P}$ mehlich $=0,02 \mathrm{cmol}_{\mathrm{c}} \mathrm{dm}^{-3} ; \mathrm{Mg}^{+2}=1,44 \mathrm{cmol}_{\mathrm{c}} \mathrm{dm}^{-3} ; \mathrm{Ca}^{+2}=0,29 \mathrm{cmol}_{\mathrm{c}} \mathrm{dm}^{-3} \mathrm{e}$ $\mathrm{Al}^{+3}=0,08 \mathrm{cmol}_{\mathrm{c}} \mathrm{dm}^{-3}$.

As mudas utilizadas foram de tomate tipo Pizzadoro para o primeiro experimento (E1) e Carina para o segundo experimento (E2), provenientes de viveiros comerciais certificados, que foram transplantadas para os vasos com 25 a $30 \mathrm{~cm}$ de altura.

O controle fitossanitário foi realizado preventivamente e foram aplicados os seguintes inseticidas desde o início do cultivo: Cipermetrina (piretróide), um $\mathrm{mL} \mathrm{L}^{-1}$, a cada 15 dias, para broca grande do fruto (Helicoverpa zea) e Bacillus thuringiensis (biológico), um $\mathrm{mL} \mathrm{L}^{-1}$, uma vez por semana, para a traça-do-tomateiro (Tuta absoluta). Os fungicidas aplicados, a partir da fase reprodutiva, foram: Clorotalonil, cinco $\mathrm{mL} \mathrm{L}^{-1}$, uma vez por semana para pintapreta, septoriose e oídio (Oidium neolycopersici), e Azoxistrobina com Difenoconazol, um $\mathrm{mL} \mathrm{L}^{-1}$, uma vez a cada 15 dias, também para pinta-preta. 
Os experimentos foram conduzidos com cinco tratamentos que consistiram em cinco concentrações de K na solução nutritiva $\left(60,120\right.$, 180, 240 e $300 \mathrm{mg} \mathrm{dm}^{-3}$ de K) aplicados após a abertura das primeiras flores, com 29 dias após o transplantio, sendo que até esse estádio de desenvolvimento, a solução nutritiva foi padrão para todos os tratamentos. Estas doses foram estabelecidas a partir de conhecimento prévio da dose média de $\mathrm{K}$ que é recomendada para a cultura do tomateiro, que seria $180 \mathrm{mg} \mathrm{dm}^{-3}$ (ALMEIDA et al., 2014).

As doses dos nutrientes foram de $\left(\mathrm{mg} \mathrm{dm}^{-3}\right)$ : N: 198; P: 43,6; K: 152,4; Ca: 233; Mg: 27 e S: 39. Foram utilizados como fertilizantes: o MAP (200 g $\left.1000 \mathrm{~L}^{-1}\right) ; \mathrm{Ca}\left(\mathrm{NO}_{3}\right)_{2}(800 \mathrm{~g} 1000$ $\left.\mathrm{L}^{-1}\right) ; \mathrm{CaCl}_{2}\left(300 \mathrm{~g} 1000 \mathrm{~L}^{-1}\right) ; \mathrm{MgSO}_{4}\left(300 \mathrm{~g} 1000 \mathrm{~L}^{-1}\right)$ e $\mathrm{KNO}_{3}\left(400 \mathrm{~g} 1000 \mathrm{~L}^{-1}\right)$. Os micronutrientes foram fornecidos por meio de Rexolin $\mathrm{BRA} \circledast\left(11,6 \% \mathrm{~K}_{2} \mathrm{O} ; 1,28 \% \mathrm{~S} ; 0,86 \%\right.$ $\mathrm{Mg} ; 2,1 \% \mathrm{~B} ; 0,36 \% \mathrm{Cu} ; 2,66 \% \mathrm{Fe} ; 2,48 \% \mathrm{Mn} ; 0,036 \%$ Mo e 3,38\% Zn) e Rexolin M48® (65\% Fe quelatizado por EDDHMA), ambos na concentração de 25 g $1000 \mathrm{~L}^{-1}$.

O monitoramento da concentração de cada nutriente presente se deu por meio da medição periódica da condutividade elétrica da solução nas caixas d'água e do eluviado resultante da solução nutritiva que passou pelo vaso e ficava retida no prato abaixo do mesmo, não deixando a condutividade do sistema ultrapassar $3 \mathrm{dS} \mathrm{m}^{-1}$ o que poderia prejudicar o desenvolvimento das plantas. Quando a condutividade ultrapassou $3 \mathrm{dS} \mathrm{m}^{-1}$ a fertirrigacão foi interrompida e o sistema irrigado por um dia apenas com água para evitar salinização do sistema.

Tabela 1. Concentração de nutrientes $\left(\mathrm{mg} \mathrm{dm}^{-3}\right)$ e condutividade elétrica $(\mathrm{CE})\left(\mathrm{dS} \mathrm{m}^{-1}\right)$ das soluções nutritivas usadas nos tratamentos. Concentration of nutrients $\left(\mathrm{mg} \mathrm{dm}^{-3}\right)$ and electrical conductivity (EC) $\left(d S \mathrm{~m}^{-1}\right)$ of the nutrient solutions used in the treatments.

Tratamentos contendo $\mathrm{K}\left(\mathrm{mg} \mathrm{dm}^{-3}\right)$ e Condutividades da Solução Nutritiva***

\begin{tabular}{cccccccc}
\hline Nutriente & $\begin{array}{c}\text { Solução } \\
\text { Completa } \\
(100 \%)^{*}\end{array}$ & $\begin{array}{c}\text { Solução* } \\
(70 \%)^{* *}\end{array}$ & $\begin{array}{c}60 \\
(2,07)^{* * *}\end{array}$ & $\begin{array}{c}120 \\
(2,14)^{* * *}\end{array}$ & $\begin{array}{c}180 \\
(2,15)^{* * *}\end{array}$ & $\begin{array}{c}240 \\
(2,26)^{* * *}\end{array}$ & $\begin{array}{c}300 \\
(2,42)^{* * *}\end{array}$ \\
\hline $\mathrm{N}$ & 198 & 138,6 & 198,0 & 198,0 & 198,0 & 198,0 & 198,0 \\
$\mathrm{P}$ & 43,6 & 30,52 & 43,6 & 43,6 & 43,6 & 43,6 & 43,6 \\
$\mathrm{~K}$ & 152,4 & 106,68 & 60,0 & 120,0 & 180,0 & 240,0 & 300,0 \\
$\mathrm{Ca}$ & 233 & 163,1 & 233,0 & 233,0 & 233,0 & 233,0 & 233,0 \\
$\mathrm{Mg}$ & 27 & 18,9 & 27 & 27 & 27 & 27 & 27 \\
$\mathrm{~S}$ & 39 & 27,3 & 39 & 39 & 39 & 39 & 39 \\
$\mathrm{~B}$ & 0,5 & 0,35 & 0,5 & 0,5 & 0,5 & 0,5 & 0,5 \\
$\mathrm{Fe}$ & 5 & 3,5 & 5 & 5 & 5 & 5 & 5 \\
$\mathrm{Cu}$ & 0,07 & 0,049 & 0,07 & 0,07 & 0,07 & 0,07 & 0,07 \\
$\mathrm{Mn}$ & 0,1 & 0,07 & 1 & 1 & 1 & 1 & 1 \\
$\mathrm{Mo}$ & 0,075 & 0,0525 & 0,075 & 0,075 & 0,075 & 0,075 & 0,075 \\
$\mathrm{Zn}$ & 0,4 & 0,28 & 0,4 & 0,4 & 0,4 & 0,4 & 0,4 \\
\hline
\end{tabular}

*Sarruge (1975) modificado e utilizado no Laboratório de Solos da UEL. **Solução nutritiva utilizada por 15 dias, para adaptação das mudas. .**Médias da condutividade elétrica (CE) da solução nutritiva medida nas caixas d'água $\left(\mathrm{dS} \mathrm{m}^{-1}\right)$. *Sarruge (1975) modified and used in the Soil Laboratory of UEL. **Nutritional solution used for 15 days for seedlings adaption. ***Means of electrical conductivity (EC) of nutrient solution measured in water boxes $\left(d S \mathrm{~m}^{-1}\right)$. 
As aplicações de fertilizantes foram realizadas via água de irrigação com a frequência variável a fim de que as perdas não ultrapassassem $10 \%$ por turno de rega, sendo que cada gotejador foi regulado para uma vazão máxima de $300 \mathrm{~mL} \mathrm{~min}^{-1}$. O turno de rega foi definido com base nas características climáticas - temperatura, umidade relativa do ar, que foram medidas dentro da casa de vegetação durante o experimento, e pelas características da cultura, variando de 1 a 5 vezes por dia o turno.

As médias mensais da temperatura durante a condução do experimento foram de 33,6 ${ }^{\circ} \mathrm{C}$ do dia 27 a 31 de março, $26{ }^{\circ} \mathrm{C}$ em abril, $27,5^{\circ} \mathrm{C}$ em maio, $23,8{ }^{\circ} \mathrm{C}$ em junho e $27,9{ }^{\circ} \mathrm{C}$ até o dia 29 de julho medidas através de datalogger da marca Instrutherm.

A umidade relativa do ar (URA) mensurada foi de $42,0 \%$ de 27 a 31 de março, 64,4\% em abril, 57,0\% em maio, 72,6\% em junho e 54,1\% até o dia 29 de julho medidas através de datalogger da marca Instrutherm.

As plantas foram conduzidas com três cachos de tomate, sendo após o terceiro cacho contadas cinco folhas e as plantas podadas para cortar a dominância apical.

Aos 69, 93 e 115 dias após transplantio (DAT), foi coletada a folha imediatamente a cada cacho para avaliar as condições nutricionais da cultura e determinar a folha índice mais representativa do tomateiro em relação ao aumento das doses de potássio.

Todo o material vegetal coletado foi acondicionado em sacos de papel tipo Kraft devidamente etiquetados e levados à estufa de circulação de ar forçado a $65^{\circ} \mathrm{C}$ até atingirem massa constante e posteriormente triturados em moinho tipo Willey.

Ao final dos experimentos foram obtidas as produtividades, contabilizando a massa fresca de todos os tomates produzidos durante o ciclo, e todo material vegetal coletado foi preparado para determinação dos nutrientes, onde por digestão sulfúrica foram obtidos os teores de N pelo método do microdestilador de Kjeldahl (BREMNER; KEENEY, 1965). A partir de digestão nítrico-perclórica foram determinados os demais nutrientes, sendo os teores de P pela redução do fosfomolibdato pelo ácido ascórbico (BRAGA; DEFELIPO, 1974), o S por turbidimetria do sulfato (BLANCHAR et al., 1965), o K por fotometria de chama e o Ca, $\mathrm{Mg}$ e micronutrientes por espectrofotometria de absorção atômica.

Os dados referentes aos teores nutricionais e a produtividade foram submetidos à analise de variância. Aos valores médios de cada tratamento foram ajustados modelos de regressão polinomial até segundo grau.

Foi feita também análise de correlação dos nutrientes com as características avaliadas. Todas as análises foram realizadas com software $\mathrm{R}$.

\section{RESULTADOS E DISCUSSÃO}

Em relação à produtividade o maior valor encontrado nos dois experimentos foi na dose de $300 \mathrm{mg} \mathrm{dm}^{-3}$ de $\mathrm{K}$, com 81,01 $\mathrm{t} \mathrm{ha}^{-1}$ para o E1 e 99,32 t ha-1 para o E2 (Figura 1), concordando com Sara Mejía et al. (2007) que também observaram incremento na produção de massa fresca de acordo com o aumento da concentração de nutrientes até que se atinja o ponto de saturação onde a produção diminui consideravelmente. No entanto, estatisticamente, 
o E1 teve ajuste quadrático, com melhor produtividade na dose de $180 \mathrm{mg} \mathrm{dm}^{-3}$ de $\mathrm{K}$ e o E2

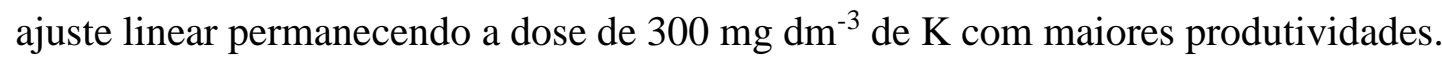

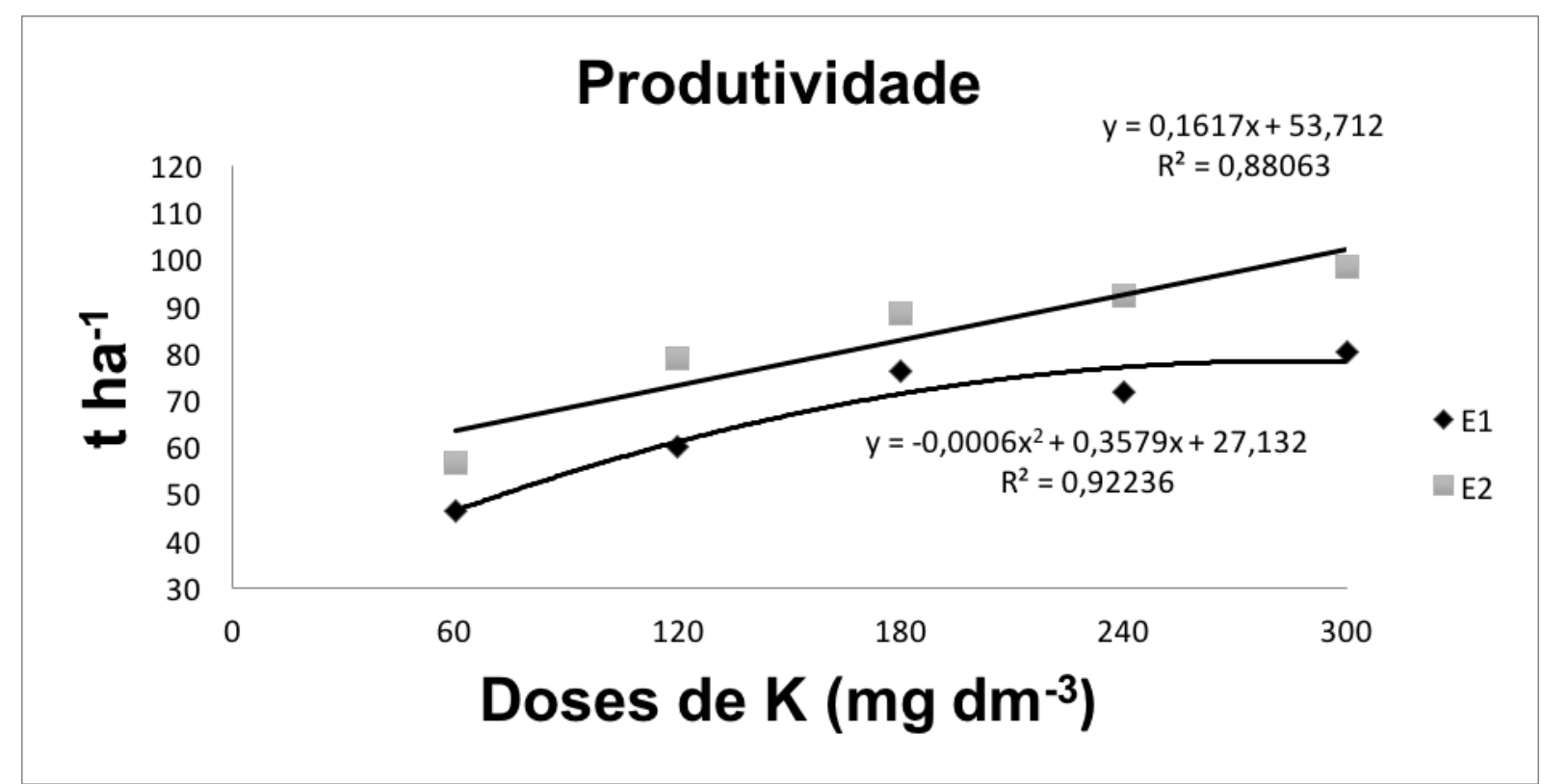

Figura 1. Médias das produtividades $\left(\mathrm{t} \mathrm{ha}^{-1}\right)$ de duas cultivares de tomateiro em função de doses de $\mathrm{K}$ na solução nutritiva dos dois experimentos. Yeild means ( $t \mathrm{ha}^{-1}$ ) of two tomato cultivars as a function of $K$ doses in the nutrient solution of the two experiments.

Cook e Sander (1991) também observaram aumento significativo na produtividade do tomateiro com o incremento nas doses de $\mathrm{K}$, concordando com os resultados do E2 que teve ajuste linear conforme o aumento das doses. E1 teve um ajuste quadrático com a tendência de maior produtividade para a dose de $180 \mathrm{mg} \mathrm{dm}^{-3}$, assim como observado por Coltman e Riede (1992) que obtiveram resultados próximos trabalhando com o tomate cultivar Celebrity cultivado sob fertirrigação em condições de casa de vegetação com cinco níveis de K, tendo maiores produtividades com a dose de $200 \mathrm{mg} \mathrm{dm}^{-3}$.

A produtividade total obtida no experimento foi semelhante à de $97,9 \mathrm{t} \mathrm{ha}^{-1}$ registrada por Macêdo (2005). Segundo Fontes et al. (2000), a produtividade comercial e total do tomateiro teria aumentado com o incremento nas doses de $\mathrm{K}$, alcançando um máximo de 73,4 e 86,4 t ha-1 , com a aplicação de 194 e $198 \mathrm{~kg} \mathrm{ha}^{-1}$ de K, respectivamente.

Já Roquejani et al. (2008), em seus estudos com produtividade e qualidade de híbridos de tomate do tipo italiano e Santa Cruz em casa de vegetação, quando raleados, tiveram uma produtividade média de 106,7 $\mathrm{t} \mathrm{ha}^{-1}$ da cultivar Giuliana.

Após as análises das folhas coletadas logo acima dos três cachos dos dois experimentos chegou-se aos resultados para os macro e micronutrientes apresentados nas Tabelas 2, 3, 4 e 5 .

A partir dos resultados obtidos é possível estabelecer um padrão nutricional para o tomateiro, principalmente em comparação com outros trabalhos. Foi possível estabelecer uma correlação entre a produtividade e os nutrientes que apresentaram diferença significativa, pela análise de regressão, conforme o aumento das doses $\mathrm{K}$ para se estabelecer a folha que melhor representa o tomateiro nutricionalmente. 
Tabela 2. Teores de macronutrientes em $\mathrm{g} \mathrm{kg}^{-1}$ das folhas logo acima do primeiro, segundo e terceiro cacho no inicio do desenvolvimento dos frutos do experimento 1, tomate Pizzadoro, juntamente com teores de referencia. Macronutrient contents in $\mathrm{g} \mathrm{kg}^{-1}$ of the leaves just above the first, second and third bunch at the beginning of the development of the fruits of experiment 1, tomato Pizzadoro, with reference contents.

\begin{tabular}{|c|c|c|c|c|c|c|c|}
\hline & Trat K & $\mathrm{N}$ & $\mathrm{P}$ & $\mathrm{K}$ & $\mathrm{Ca}$ & $\mathrm{Mg}$ & $S$ \\
\hline \multirow{8}{*}{$1^{\circ} \mathrm{Cacho}$} & $\mathrm{mg} \mathrm{dm}^{-3}$ & \multicolumn{6}{|c|}{$\mathrm{g} \mathrm{kg}^{-1}$} \\
\hline & 60 & 32,81 & 13,34 & 27,64 & 40,49 & 5,04 & 16,24 \\
\hline & 120 & 35,87 & 15,97 & 50,25 & 38,69 & 5,25 & 18,96 \\
\hline & 180 & 34,59 & 12,24 & 52,05 & 32,70 & 5,17 & 19,38 \\
\hline & 240 & 33,16 & 14,23 & 63,12 & 20,71 & 5,37 & 23,38 \\
\hline & 300 & 32,03 & 12,62 & 65,08 & 19,96 & 5,05 & 23,99 \\
\hline & $\mathrm{CV}(\%)$ & 5,07 & 20,30 & 17,48 & 30,79 & 25,20 & 6,51 \\
\hline & $p>F^{*}$ & $*$ & $*$ & $*$ & $*$ & ns & $*$ \\
\hline \multirow{7}{*}{$2^{\circ}$ Cacho } & 60 & 33,83 & 9,77 & 30,97 & 34,67 & 3,00 & 11,87 \\
\hline & 120 & 32,17 & 10,63 & 35,01 & 36,54 & 3,05 & 14,95 \\
\hline & 180 & 29,82 & 10,68 & 37,69 & 30,12 & 2,71 & 14,41 \\
\hline & 240 & 32,28 & 10,55 & 48,84 & 19,27 & 2,93 & 16,43 \\
\hline & 300 & 34,30 & 9,57 & 48,41 & 18,76 & 2,73 & 14,65 \\
\hline & $\mathrm{CV}(\%)$ & 14,32 & 13,76 & 25,17 & 32,66 & 21,21 & 23,42 \\
\hline & $p>F^{*}$ & ns & ns & $*$ & $*$ & ns & ns \\
\hline \multirow{11}{*}{$3^{\circ} \mathrm{Cacho}$} & 60 & 34,59 & 16,71 & 27,73 & 33,83 & 3,22 & 19,48 \\
\hline & 120 & 36,39 & 15,32 & 49,69 & 36,10 & 3,13 & 17,05 \\
\hline & 180 & 32,71 & 11,13 & 56,04 & 28,83 & 3,09 & 18,78 \\
\hline & 240 & 31,81 & 11,62 & 61,09 & 18,26 & 2,90 & 21,07 \\
\hline & 300 & 31,35 & 11,33 & 83,50 & 17,59 & 2,78 & 22,89 \\
\hline & $\mathrm{CV}(\%)$ & 5,49 & 24,53 & 11,93 & 32,49 & 13,91 & 10,99 \\
\hline & $\mathrm{p}>\mathrm{F}^{*}$ & $*$ & $*$ & $*$ & $*$ & ns & $*$ \\
\hline & 1 & 56 & 3,1 & $47-70$ & 31,6 & 8,4 & 9,8 \\
\hline & 2 & $26-40$ & 5,9 & $91-80$ & 27,4 & 4,9 & - \\
\hline & 3 & $40-60$ & $4-8$ & $30-50$ & $14-40$ & $4-8$ & $3-10$ \\
\hline & 4 & 32 & 13 & 51 & 45 & 9 & 18 \\
\hline
\end{tabular}

* Para dados significativos a 5\% pelo teste de regressão. ns: Para dados não significativos a 5\% pelo teste de regressão. 1. Areia, $9 \mathrm{dm}^{3}$ por planta, folha oposta ao $3^{\circ}$ cacho, indeterminado (FONTES et al., 2002). 2. Pecíolo da folha oposta ao $3^{\circ}$ cacho no florescimento (RIBEIRO et al., 1999). 3. Folha com pecíolo, por ocasião do $1^{\circ}$ fruto maduro: 25 plantas (RAIJ et al., 1996). 4. Hidroponia, único cacho, folha abaixo do $1^{\circ}$ cacho na fase $1^{\circ}$ fruto maduro (FERNANDES et al., 2002). *For significant data at 5\% by the regression test. ns: For data not significant at $5 \%$ by the regression test. 1 . Sand, $9 \mathrm{dm} 3$ per plant, leaf opposite the $3 \mathrm{rd}$ bunch, indeterminate (FONTES et al., 2002). 2. Petiole of the leaf opposite the 3rd bunch in flowering (RIBEIRO et al., 1999). 3. Leaf with petiole, on the occasion of the first ripe fruit: 25 plants (RAIJ et al., 1996). 4. Hydroponics, single bunch, leaf below the 1st bunch in phase 1 mature fruit (FERNANDES et al., 2002). 
Tabela 3. Teores de micronutrientes em $\mathrm{mg} \mathrm{kg}^{-1}$ das folhas logo acima do primeiro, segundo e terceiro cacho no inicio do desenvolvimento dos frutos do experimento 1, tomate Pizzadoro, juntamente com teores de referencia. Micronutrient contents in $\mathrm{mg} \mathrm{kg}^{-1}$ of the leaves just above the first, second and third bunch at the beginning of the development of the fruits of experiment 1, Pizzadoro tomato, with reference contents.

\begin{tabular}{|c|c|c|c|c|c|c|}
\hline & & Trat K & $\mathrm{Cu}$ & $\mathrm{Fe}$ & $\mathrm{Mn}$ & $\mathrm{Zn}$ \\
\hline & & $\mathrm{mg} \mathrm{dm}^{-3}$ & & & $\mathrm{mg} \mathrm{kg}^{-1}$ & \\
\hline \multirow{7}{*}{$1^{\circ} \mathrm{Cacho}$} & & 60 & 21,65 & 474,37 & 568,32 & 78,85 \\
\hline & & 120 & 19,08 & 500,87 & 578,26 & 56,83 \\
\hline & & 180 & 18,83 & 403,76 & 578,00 & 37,81 \\
\hline & & 240 & 20,58 & 445,69 & 726,49 & 75,02 \\
\hline & & 300 & 19,02 & 395,54 & 786,93 & 76,62 \\
\hline & & $\mathrm{CV}(\%)$ & 22,25 & 24,54 & 12,04 & 12,85 \\
\hline & & $\mathrm{p}>\mathrm{F}^{*}$ & $\mathrm{~ns}$ & $\mathrm{~ns}$ & $*$ & $*$ \\
\hline \multirow{7}{*}{$2^{\circ} \mathrm{Cacho}$} & & 60 & 21,11 & 480,89 & 655,78 & 38,76 \\
\hline & & 120 & 24,75 & 511,55 & 693,80 & 40,50 \\
\hline & & 180 & 28,55 & 556,70 & 697,06 & 35,70 \\
\hline & & 240 & 41,33 & 542,06 & 900,83 & 49,94 \\
\hline & & 300 & 40,39 & 608,33 & 839,39 & 48,56 \\
\hline & & $\mathrm{CV}(\%)$ & 28,83 & 16,91 & 22,43 & 32,76 \\
\hline & & $\mathrm{p}>\mathrm{F}^{*}$ & $*$ & ns & $*$ & ns \\
\hline \multirow{7}{*}{$3^{\circ} \mathrm{Cacho}$} & & 60 & 8,63 & 368,31 & 796,51 & 48,80 \\
\hline & & 120 & 10,52 & 417,39 & 619,11 & 38,60 \\
\hline & & 180 & 12,05 & 419,81 & 599,08 & 30,32 \\
\hline & & 240 & 14,38 & 279,48 & 775,85 & 61,73 \\
\hline & & 300 & 15,77 & 295,88 & 731,96 & 46,48 \\
\hline & & $\mathrm{CV}(\%)$ & 13,41 & 41,03 & 20,72 & 23,65 \\
\hline & & $\mathrm{p}>\mathrm{F}^{*}$ & $*$ & ns & $*$ & $*$ \\
\hline \multicolumn{3}{|c|}{1} & 798 & 183 & 258 & 25 \\
\hline \multicolumn{3}{|c|}{2} & 41 & 66 & 103 & 134 \\
\hline \multicolumn{3}{|c|}{3} & $5-15$ & $100-300$ & $50-250$ & $30-100$ \\
\hline \multicolumn{3}{|c|}{4} & 10 & 209 & 665 & 96 \\
\hline
\end{tabular}

*Para dados significativos a 5\% pelo teste de regressão. ns: Para dados não significativos a 5\% pelo teste de regressão. 1. Areia, $9 \mathrm{dm}^{3}$ por planta, folha oposta ao $3^{\circ}$ cacho, indeterminado (FONTES et al., 2002). 2. Pecíolo da folha oposta ao $3^{\circ}$ cacho no florescimento (RIBEIRO et al., 1999). 3. Folha com pecíolo, por ocasião do $1^{\circ}$ fruto maduro: 25 plantas (RAIJ et al., 1996). 4. Hidroponia, único cacho, folha abaixo do $1^{\circ}$ cacho na fase $1^{\circ}$ fruto maduro (FERNANDES et al., 2002). *For significant data at 5\% by the regression test. ns: For data not significant at $5 \%$ by the regression test. 1. Sand, $9 \mathrm{dm} 3$ per plant, leaf opposite the 3rd bunch, indeterminate (FONTES et al., 2002). 2. Petiole of the leaf opposite the 3rd bunch in flowering (RIBEIRO et al., 1999). 3. Leaf with petiole, on the occasion of the first ripe fruit: 25 plants (RAIJ et al., 1996). 4. Hydroponics, single bunch, leaf below the 1st bunch in phase 1 mature fruit (FERNANDES et al., 2002). 
Tabela 4. Teores de macronutrientes em $\mathrm{g} \mathrm{kg}^{-1}$ das folhas logo acima do primeiro, segundo e terceiro cacho no inicio do desenvolvimento dos frutos do experimento 2, tomate Carina, juntamente com teores de referencia. Macronutrient contents in $\mathrm{g} \mathrm{kg-1} \mathrm{of} \mathrm{the} \mathrm{leaves} \mathrm{just} \mathrm{above}$ the first, second and third bunch at the beginning of the development of the fruits of experiment 2, Carina tomato, together with reference contents.

\begin{tabular}{|c|c|c|c|c|c|c|c|}
\hline & Trat K & $\mathrm{N}$ & $\mathrm{P}$ & $\mathrm{K}$ & $\mathrm{Ca}$ & $\mathrm{Mg}$ & $S$ \\
\hline \multirow{8}{*}{$1^{\circ} \mathrm{Cacho}$} & $\mathrm{mg} \mathrm{dm}^{-3}$ & \multicolumn{6}{|c|}{$\mathrm{kg} \mathrm{ha}^{-1 * 2}$} \\
\hline & 60 & 34,47 & 11,27 & 46,39 & 40,45 & 4,51 & 11,62 \\
\hline & 120 & 29,69 & 18,11 & 50,66 & 38,66 & 5,40 & 21,13 \\
\hline & 180 & 36,70 & 11,95 & 62,53 & 34,86 & 5,12 & 20,42 \\
\hline & 240 & 34,99 & 14,15 & 63,06 & 24,73 & 5,33 & 23,29 \\
\hline & 300 & 34,22 & 12,83 & 64,92 & 19,94 & 5,01 & 21,33 \\
\hline & $\mathrm{CV}(\%)$ & 7,71 & 25,06 & 18,52 & 34,28 & 24,67 & 13,24 \\
\hline & $\mathrm{p}>\mathrm{F}^{*}$ & $*$ & $*$ & $*$ & $*$ & ns & $*$ \\
\hline \multirow{7}{*}{$2^{\circ} \mathrm{Cacho}$} & 60 & 43,00 & 12,44 & 43,52 & 33,51 & 2,71 & 6,66 \\
\hline & 120 & 37,49 & 10,02 & 65,55 & 36,50 & 3,01 & 12,42 \\
\hline & 180 & 27,20 & 11,27 & 71,35 & 31,90 & 2,35 & 12,26 \\
\hline & 240 & 32,34 & 10,21 & 73,39 & 20,41 & 2,77 & 17,70 \\
\hline & 300 & 31,89 & 9,68 & 75,50 & 18,74 & 2,88 & 18,57 \\
\hline & $\mathrm{CV}(\%)$ & 10,70 & 27,78 & 16,50 & 32,88 & 26,38 & 24,06 \\
\hline & $\mathrm{p}>\mathrm{F}^{*}$ & $*$ & ns & $*$ & * & ns & $*$ \\
\hline \multirow{7}{*}{$3^{\circ} \mathrm{Cacho}$} & 60 & 36,53 & 11,37 & 25,87 & 33,79 & 2,39 & 12,59 \\
\hline & 120 & 31,75 & 10,97 & 51,21 & 36,06 & 2,85 & 17,27 \\
\hline & 180 & 31,24 & 11,60 & 63,81 & 27,64 & 2,77 & 23,28 \\
\hline & 240 & 30,23 & 9,78 & 69,17 & 18,21 & 2,97 & 20,41 \\
\hline & 300 & 34,82 & 11,20 & 72,15 & 17,57 & 2,96 & 23,05 \\
\hline & $\mathrm{CV}(\%)$ & 6,65 & 14,23 & 14,62 & 30,21 & 18,82 & 13,46 \\
\hline & $\mathrm{p}>\mathrm{F}^{*}$ & $*$ & ns & $*$ & $*$ & ns & $*$ \\
\hline \multicolumn{2}{|c|}{1} & 56 & 3,1 & $47-70$ & 31,6 & 8,4 & 9,8 \\
\hline \multicolumn{2}{|c|}{2} & $26-40$ & 5,9 & $91-80$ & 27,4 & 4,9 & - \\
\hline \multicolumn{2}{|c|}{3} & $40-60$ & $4-8$ & $30-50$ & $14-40$ & $4-8$ & $3-10$ \\
\hline \multicolumn{2}{|c|}{4} & 32 & 13 & 51 & 45 & 9 & 18 \\
\hline
\end{tabular}

*Para dados significativos a 5\% pelo teste de regressão. ns: Para dados não significativos a 5\% pelo teste de regressão. 1. Areia, $9 \mathrm{dm}^{3}$ por planta, folha oposta ao $3^{\circ}$ cacho, indeterminado (FONTES et al., 2002). 2. Pecíolo da folha oposta ao $3^{\circ}$ cacho no florescimento (RIBEIRO et al., 1999). 3. Folha com pecíolo, por ocasião do $1^{\circ}$ fruto maduro: 25 plantas (RAIJ et al., 1996). 4. Hidroponia, único cacho, folha abaixo do $1^{\circ}$ cacho na fase $1^{\circ}$ fruto maduro (FERNANDES et al., 2002). *For significant data at $5 \%$ by the regression test. ns: For data not significant at $5 \%$ by the regression test. 1. Sand, $9 \mathrm{dm} 3$ per plant, leaf opposite the $3 \mathrm{rd}$ bunch, indeterminate (FONTES et al., 2002). 2. Petiole of the leaf opposite the 3rd bunch in flowering (RIBEIRO et al., 1999). 3. Leaf with petiole, on the occasion of the first ripe fruit: 25 plants (RAIJ et al., 1996). 4. Hydroponics, single bunch, leaf below the 1st bunch in phase 1 mature fruit (FERNANDES et al., 2002). 
Tabela 5. Teores de micronutrientes em $\mathrm{mg} \mathrm{kg}^{-1}$ das folhas logo acima do primeiro, segundo e terceiro cacho no inicio do desenvolvimento dos frutos do experimento 2, tomate Carina, juntamente com teores de referencia. Micronutrient contents in $\mathrm{mg} \mathrm{kg}^{-1}$ of the leaves just above the first, second and third bunch at the beginning of the development of the fruits of experiment 2, Carina tomato, with reference contents.

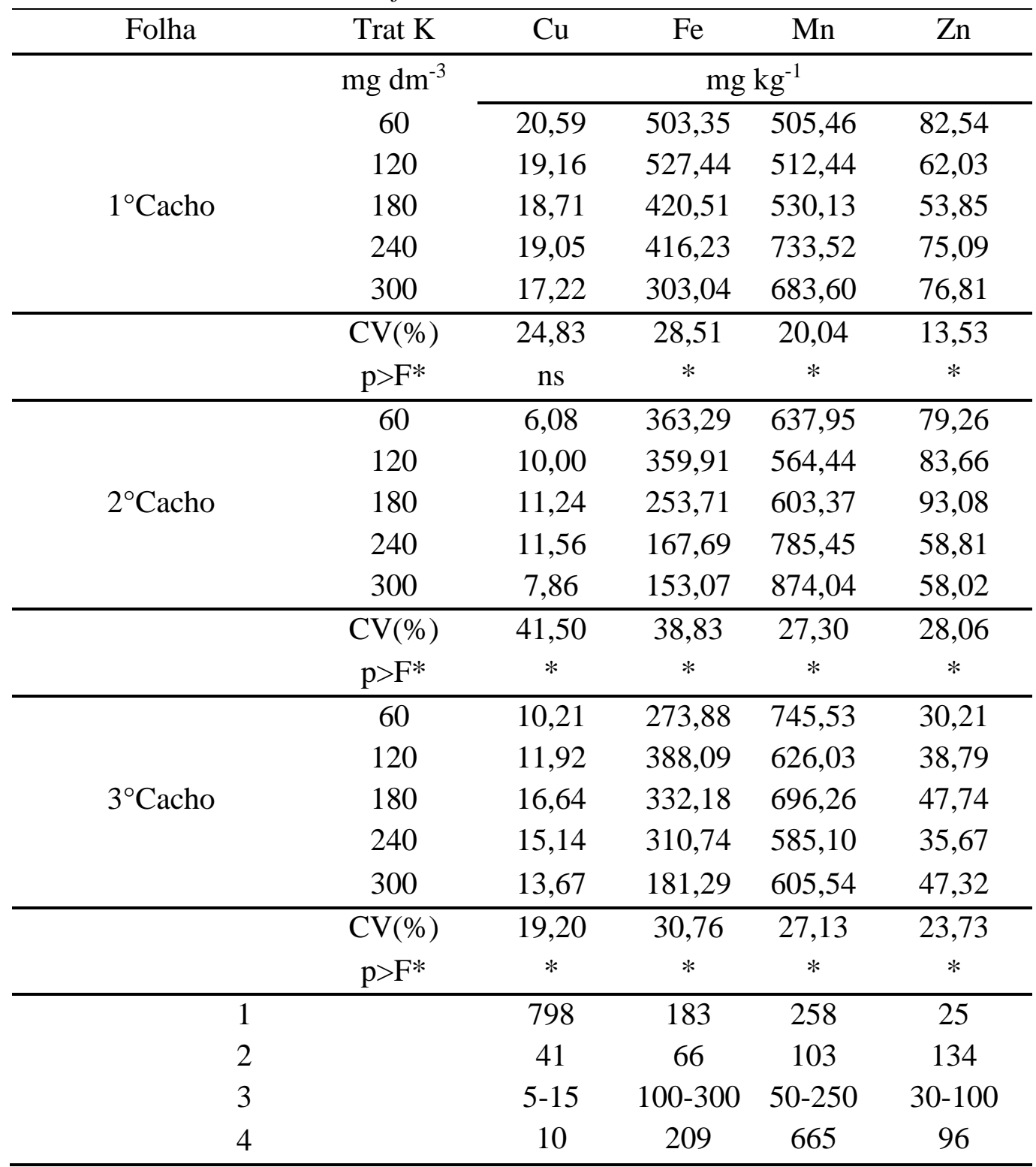

*Para dados significativos a 5\% pelo teste de regressão. ns: Para dados não significativos a 5\% pelo teste de regressão. 1. Areia, $9 \mathrm{dm}^{3}$ por planta, folha oposta ao $3^{\circ}$ cacho, indeterminado (FONTES et al., 2002). 2. Pecíolo da folha oposta ao $3^{\circ}$ cacho no florescimento (RIBEIRO et al., 1999). 3. Folha com pecíolo, por ocasião do $1^{\circ}$ fruto maduro: 25 plantas (RAIJ et al., 1996). 4. Hidroponia, único cacho, folha abaixo do $1^{\circ}$ cacho na fase $1^{\circ}$ fruto maduro (FERNANDES et al., 2002). *For significant data at 5\% by the regression test. ns: For data not significant at $5 \%$ by the regression test. 1. Sand, $9 \mathrm{dm} 3$ per plant, leaf opposite the $3 \mathrm{rd}$ bunch, indeterminate (FONTES et al., 2002). 2. Petiole of the leaf opposite the 3rd bunch in flowering (RIBEIRO et al., 1999). 3. Leaf with petiole, on the occasion of the first ripe fruit: 25 plants (RAIJ et al., 1996). 4. Hydroponics, single bunch, leaf below the 1st bunch in phase 1 mature fruit (FERNANDES et al., 2002).

Para os teores nutricionais das folhas índice encontradas acima de cada cacho no E1, cultivado com tomate Pizzadoro, encontrou-se conforme o aumento das doses de $\mathrm{K}$ uma diminuição nos teores de $\mathrm{N}$ e de $\mathrm{Ca}$ e aumento quadrático dos teores de $\mathrm{P}$. Houve aumento 
nos teores de $\mathrm{S}$ e de $\mathrm{Mn}$ (Tabelas 2 e 3 ). Os valores encontrados nas folhas acima do primeiro cacho concordam com os encontrados por Fernandes et al. (2002).

Nas folhas coletadas acima do segundo cacho (Tabelas 2 e 3) houve um aumento nos teores de $\mathrm{N}$, de $\mathrm{K}, \mathrm{Cu}$ e $\mathrm{Mn}$ e diminuição dos teores de $\mathrm{Ca}\left(34,67\right.$ a $\left.18,76 \mathrm{~g} \mathrm{~kg}^{-1}\right)$, concordando com Fernandes et al. (2002) para N, P e S e com Raij et al. (1996) para os outros nutrientes analisados.

As folhas índice acima do terceiro cacho (Tabelas 2 e 3) apresentaram uma diminuição no teor de N, P, Ca e Mn e aumento nos teores de K, S e Cu, concordando com Fontes et al. (2002), Ribeiro et al. (1999) e Fernandes et al. (2002).

No E2, cultivado com tomate Carina, quando analisados os teores nutricionais da folha índice acima do primeiro cacho observou-se uma relação quadrática conforme o aumento das doses de $\mathrm{K}$ para o $\mathrm{N}$ e para o $\mathrm{P}$ (Tabelas 4 e 5). Houve um aumento nos teores de $\mathrm{K}$ e diminuição dos teores de $\mathrm{Ca}$, conforme o aumento das doses de $\mathrm{K}$, concordando com Fernandes et al. (2002).

Para as folhas do segundo cacho (Tabelas 4 e 5) houve uma redução nos teores de N, $\mathrm{Ca}, \mathrm{Fe}$, aumento nos teores de $\mathrm{K}, \mathrm{S}$ e $\mathrm{Mn}$ e resposta quadrática para $\mathrm{o} \mathrm{Cu}$. Os resultados concordam com Fernandes et al. (2002) para N, P e S e com Raij et al. (1996) para os demais nutrientes analisados.

Quando analisadas as folhas logo acima do terceiro (Tabelas 4 e 5) cacho observou-se diminuição nos teores de $\mathrm{N}$ e Mn e aumento nos teores de K, S. Houve resposta quadrática para $\mathrm{Ca}$ e Fe. Os resultados concordam com Fontes et al. (2002) nos teores de K e Ca, com Ribeiro et al. (1999), que trabalharam em análises com folha e pecíolo opostos ao terceiro cacho, em N e Mg e Fernandes et al. (2002) para os demais nutrientes.

Analisando em conjunto o comportamento dos nutrientes nos dois experimentos nas folhas índice acima dos três cachos, conforme o aumento das doses de $\mathrm{K}$ verificou-se um maior teor de $\mathrm{Mn}$ nas folhas do primeiro e segundo cacho dos dois experimentos e diminuição nas folhas do terceiro cacho, sendo este comportamento atribuído principalmente pelo excesso de K provocar deficiência de Mn, conforme constatado por Silva et al. (1995) e Carvalho et al. (2001) em trabalho com aumento de doses de K em maracujazeiro.

Nas folhas acima de todos os cachos nos dois experimentos também verificou-se redução nos teores de Ca conforme o aumento das doses de $\mathrm{K}$, sendo mais um caso de competição entre cátions, verificado desde o inicio de desenvolvimento da planta concordando com os relatos de Mascarenhas et al. (2000) e Oliveira et al. (2001).

Segundo Malavolta et al. (2006) conforme aumento dos teores de K na solução nutritiva há competição na absorção do $\mathrm{Ca}$ e $\mathrm{Mg}$, pois, durante o processo de absorção, estes nutrientes utilizaram os mesmos sítios carregadores.

Nos dois experimentos o teor de $\mathrm{P}$ teve resposta quadrática nas folhas do primeiro cacho e o teor de $\mathrm{Cu}$ aumentou nas folhas do segundo e terceiro cacho do E1, tendo comportamento quadrático nas folhas dos mesmos cachos no E2, conforme o aumento de doses de $\mathrm{K}$, fato relacionado com a diminuição dos teores de $\mathrm{Ca}$ que em aumento diminui os teores de $\mathrm{Cu}$. 
Observou-se também uma diminuição nos teores de $\mathrm{N}$ nas folhas acima do terceiro cacho dos dois experimentos atribuída ao aumento de doses de $\mathrm{K}$ que induz um aumento na síntese de proteínas na planta consumindo o N. Pelo aumento da síntese de proteínas também observou-se um aumento no teor de $\mathrm{S}$ nas folhas índice, acima dos três cachos, nos dois experimentos, resultado da alta necessidade deste macronutriente acarretada pelo aumento das doses de $\mathrm{K}$.

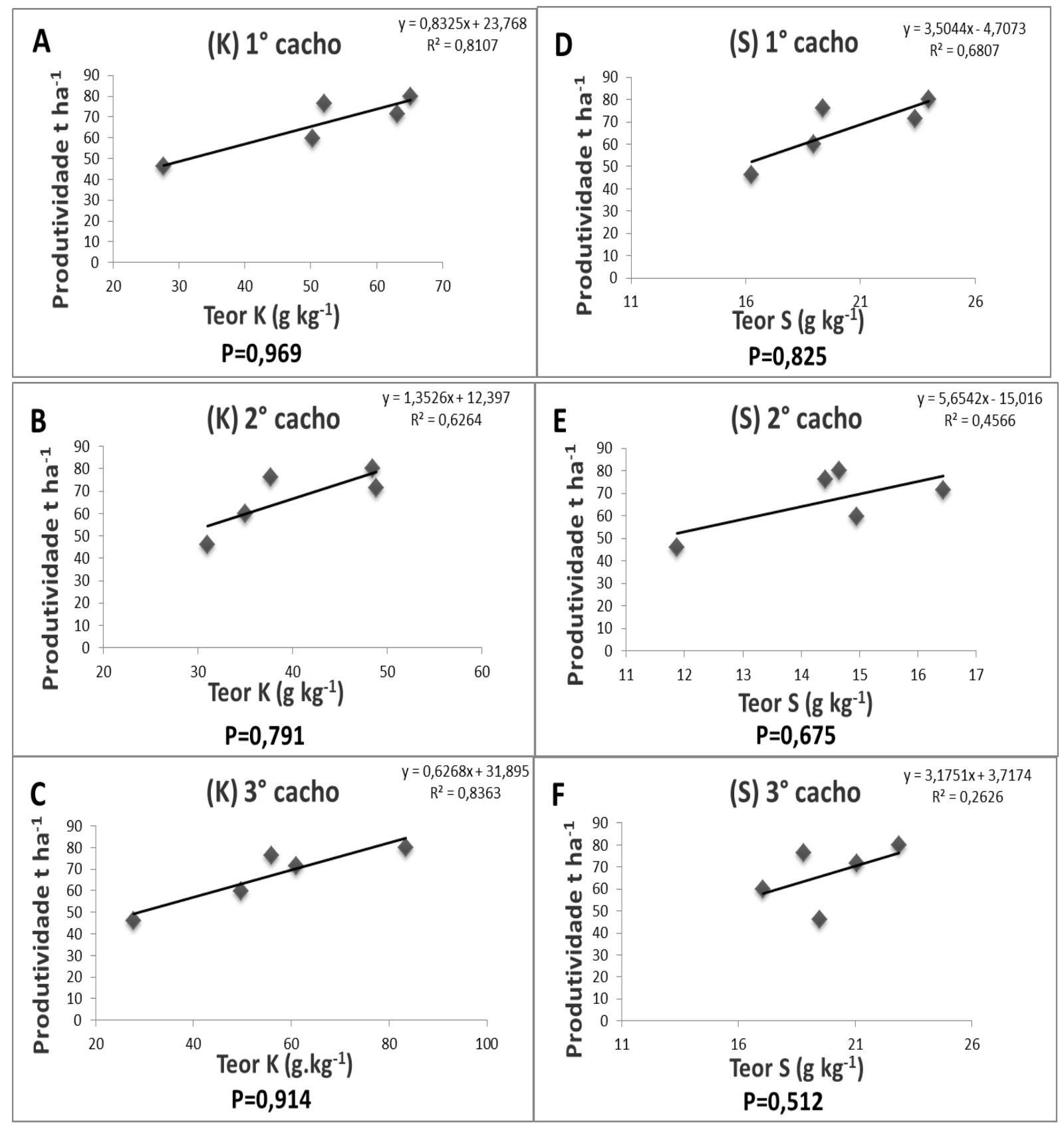

Figura 2. Relação entre os teores de $\mathrm{K}$ e $\mathrm{S}$ encontrados nas folhas acima dos três cachos com a produtividade no E1 com variedade Pizzadoro. Relationship between the contents of $K$ and $S$ found in leaves above the three bunches with yeild in E1 with Pizzadoro variety.

Mesmo verificando um inicio de competição entre os cátions conforme o aumento das doses, os tratamentos não prejudicaram a produção, tendo influencia positiva no aumento de síntese de proteínas e produtividade nas duas cultivares. 
Foram comparados o teor de nutrientes nas folhas acima dos três cachos para se verificar a que melhor representa nutricionalmente o tomateiro. Assim foi correlacionado o aumento dos teores nutricionais, significativos por análise da regressão, com a produção.

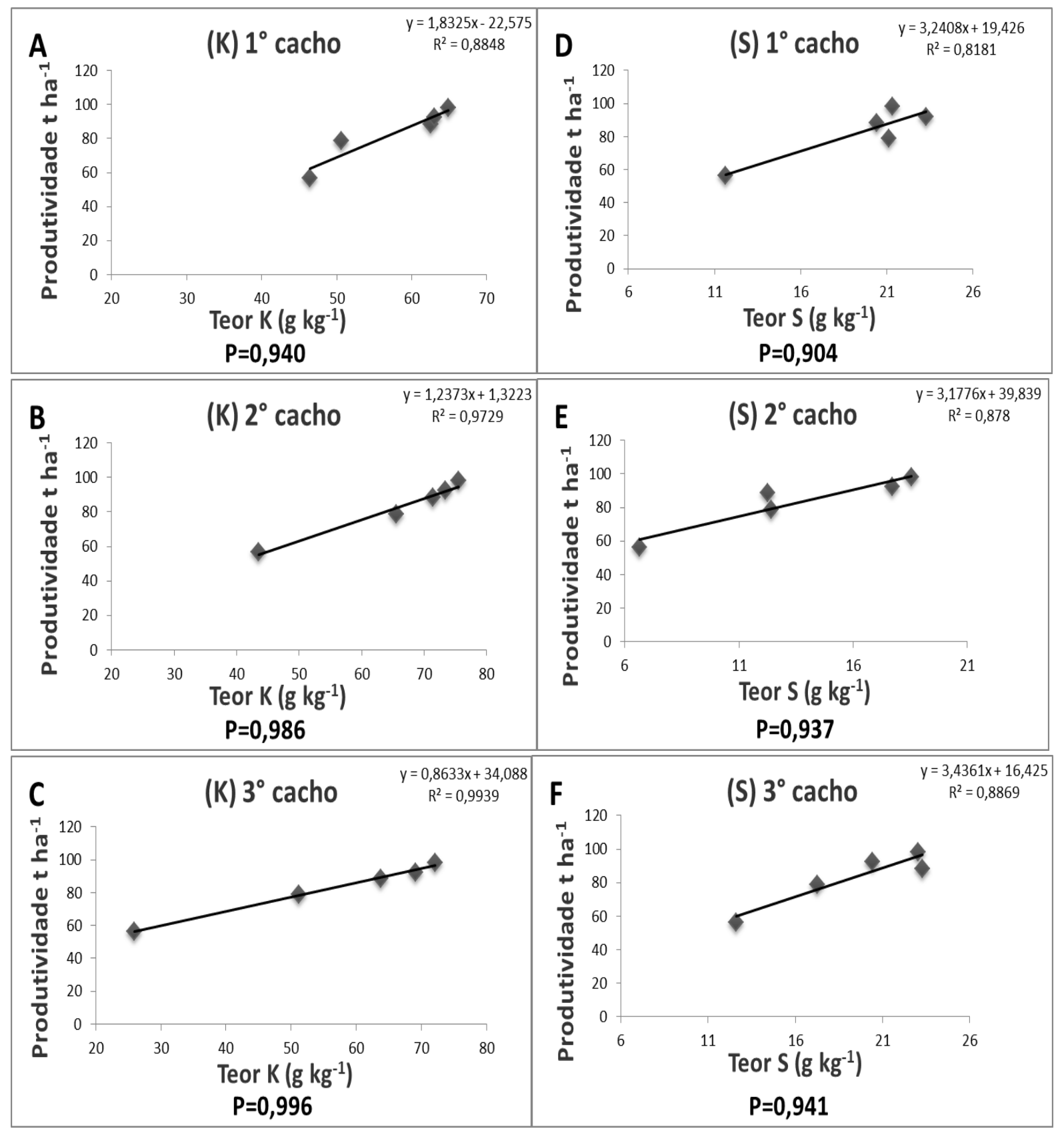

Figura 3. Relação entre os teores de Potássio e Enxofre encontrados nas folhas acima dos três cachos com a produtividade. Relation between Potassium and Sulfur levels found in leaves above the three bunches with yield.

Após as correlações entre a produção e os teores significativos nas folhas acima dos três cachos no E1 para K obteve-se uma correlação de 0,969 no primeiro cacho (Figura 2A), 0,791 no segundo (Figura 2B) e 0,914 no terceiro cacho (Figura 2C). Para os teores de S chegou-se a 0,825 (Figura 2D) nas folhas acima do primeiro cacho, 0,675 no segundo (Figura 2E) e 0,512 no terceiro cacho (Figura $2 \mathrm{~F}$ ), concluindo que no E1 com a variedade Pizzadoro a folha 
que melhor representa o tomateiro visando à produção é a que fica logo acima do primeiro cacho.

Para o E2 a folha acima do terceiro cacho foi a que melhor representou o tomateiro nutricionalmente, visando à produção, onde comparando esta com teores de K obteve-se uma correlação de 0,940 para as folhas acima do primeiro cacho (Figura 3A), 0,986 para o segundo cacho (Figura 3B) e 0,996 para as folhas acima do terceiro cacho (Figura 3C). Para os teores de $\mathrm{S}$ obteve-se uma correlação positiva de 0,904 entre as folhas acima do primeiro cacho (Figura 3D), 0,937 para o segundo cacho e 0,941 (Figura 3E) para o terceiro cacho (Figura 3F).

Os resultados da folha índice mais representativa variaram de acordo com a variedade utilizada, sendo para o tomate Pizzadoro (E1), tipo italiano, a folha acima do primeiro cacho e para o tomate Carina (E2), grupo santa cruz, a folha acima do terceiro cacho, porém de acordo com os resultados as correlações entre o teor nutricional das folhas e a produção sempre foram maiores que 0,800 , demonstrando que para cultivar ainda não estudadas folhas acima de qualquer cacho podem ser usadas com boa correlação.

\section{CONCLUSÃO}

O aumento das doses de potássio influenciou significativamente a produtividade e o teor nutricional no tecido vegetal.

No E1 a folha que melhor representa o tomateiro nutricionalmente visando à produção é a que fica logo acima do primeiro cacho, já para o E2 é a folha acima do terceiro cacho.

Para cultivares ainda não estudadas, folhas acima de qualquer cacho podem ser utilizadas com boa correlação.

\section{REFERÊNCIAS BIBLIOGRÁFICAS}

ALMEIDA, L. H. C.; OLIVEIRA, E. C.; FRATONI, M. M. J; FREGONEZI, G. A. F.; TAKAHASHI, H. W. Effect of potassium doses fertirrigated in the nutritional contents of tomato fruit and leaves in their early development. African journal of agriculture, New York, v. 10, n. 1, p.4275-4282, 2015.

ALMEIDA, L. H. C.; FRATONI, M. M. J.; FREGONEZI, G. A. F.; TAKAHASHI, H. W. Nutritional assessment of potassium in tomato (Lycopersicon esculentum Mill.) by direct reading of fruit sap. African Journal of Agricultural Research, Nairobi, v. 11, n. 14, p.1245-1256, 2016.

ANDRIOLO, J. L.; DUARTE, T. S.; LUDKE, L.; SKREBSKY, E. C. Crescimento e desenvolvimento do tomateiro cultivado em substrato com fertirrigação. Horticultura brasileira, Brasília, v. 15, n. 1, p.28-32, 1997.

ANDRIOLO, J. L.; LUZ, G. L. DA.; BORTOLOTTO, O. C.; GODOI, R. DOS S. Produtividade e qualidade de frutos de meloeiro cultivado em substrato com três doses de solução nutritiva. Ciência Rural, Santa Maria, v. 35, n. 4, p.781-787, 2005. 
AYRES, R. S; WESTCOT, D. W. A qualidade da água na agricultura. Water Quality for Agriculture. Paraíba: UFPB. 1991. 218 p.

BLANCHAR, R. W.; REHM, G.; CALDWELL, A. C. Sulfur in plant material by digestion with nitric and perchloric acid. Soil Science Society of America, Madison, v. 29, n. 1, p.71$72,1965$.

BRAGA, G. N. M. As vantagens da Fertirrigação. 2010. Disponível em: <http://agronomiacomgismonti.blogspot.com.br/2010/04/as-vantagens-dafertirrigacao.html>. Acesso em: 30 mai. 2014.

BRAGA, J. M.; DEFELIPO, B. Determinação espectrofotométrica de fósforo em extratos de solos e plantas. Revista Ceres, Viçosa, v. 21, n. 113, p.73-85, 1974.

BREMNER, J. M.; KEENEY, D. R. Determination and isotope-ratio analysis of different forms of nitrogen in soils 3: Exchangeable ammonium, nitrate, and nitrite by extractiondistillation methods. Soil Science Society of America Journal, Madison, v. 30, n. 5, p.577$582,1965$.

CARVAlHO, A. J. C.; MARTINS, D. P.; MONNERAT, P. H.; BERNARDO, S.; SILVA, J. A. Teores de nutrientes foliares no maracujazeiro-amarelo associados à estação fenológica, adubação potássica e lâminas de irrigação. Revista Brasileira de Fruticultura, Jaboticabal, v. 23, n. 2, p.403-408, 2001.

COLTMAN, R. R.; RIEDE, S. A. Monitoring the potassium status of greenhouse tomatoes using quick petiole sap tests. Hortscience, Alexandria, v. 27, n. 4, p.361-364, 1992.

COOK, W. P.; SANDERS, D. C. Nitrogen application frequency for drip-irrigated tomatoes. HortScience, Mont Vernon, v. 26, n. 3, p.250-252, 1991.

FAO. Food and Agriculture Organization of the United Nations. 2015. Disponível em <http://www.fao.org/brasil/parceiros/pt/>. Acesso em: 05 abr. 2017.

FAQUIN, V. Nutrição mineral de plantas. Lavras: UFLA/FAEPE, 2001. 182 p.

FERNANDES, A. A.; MARTINEZ, H. E. P.; FONTES, P. C. R. Produtividade, qualidade dos frutos e estado nutricional do tomateiro tipo longa vida conduzido com um cacho, em cultivo hidropônico, em função das fontes de nutrientes. Horticultura Brasileira, Brasília, v. 20, n. 4, p.564-570, 2002.

FERNANDES, C.; ARAÚJO, J. A. C.; CORÁ, J. E. Impacto de quatro substratos e parcelamento da fertirrigação na produção de tomate sob cultivo protegido. Horticultura Brasileira, Brasília, v. 20, n. 4, p.559-563, 2002.

FONTES, P. C. R.; SILVA, D. J. H. Doenças e pragas: é seguro comer tomate? In: FONTES, P. C. R.; SILVA, D. J. H. Produção de tomate de mesa. Viçosa: UFV, 2002. cap. 20 , p. 97 129.

FONTES, P. C. R.; SAMPAIO, R. A.; MANTOVANI, E. C. Tomato yield and potassium concentrations in soil and in plant petioles as affected by potassium fertigation, Pesquisa Agropecuária Brasileira, Brasília, v. 35, n. 3, p.575-580, 2000. 
KAMALULDEENA, J.; YUNUSA, I. A. M.; ZERIHUN, A; BRUHL, J. J.; KRISTIANSEN, P. Uptake and distribution of ions reveal contrasting tolerancemechanisms for soil and water salinity in okra (Abelmoschus esculentus) and tomato (Solanum esculentum). Agricultural Water Management, Netherlands, v. 146, n. 7, p.95-104, 2014.

MACÊDO, L. S. Effects of water levels and potassium fertirrigation on growth, production and quality of tomato fruits in greenhouse. Ciência e Agrotecnologia, Lavras, v. 29, n. 2, p.296-304, 2005.

MALAVOLTA, E. Manual de nutrição mineral de plantas. Piracicaba: CERES, 2006. 631 p.

MARSCHNER, H. Mineral nutrition of higher plants. 2. ed. London: Academic Press, 1995. 889 p.

MASCARENHAS, H. A. A.; TANAKA, R. T.; CARMELLO, Q. A. C. Calcário e potássio para a cultura de soja. Scientia Agricola, Piracicaba, v. 57, n. 3, p.445-449, 2000.

OLIVEIRA, F. A.; CARMELLO, Q. A. C.; MASCARENHAS, H.A.A. Disponibilidade de potássio e suas relações com cálcio e magnésio em soja cultivada em casa de vegetação. Scientia Agricola, Piracicaba, v. 58, n. 2, p.329-335, 2001.

RAIJ, B.; CANTARELlA, H.; QUAGGIO, J. A.; FURLANI, A. M. C. Recomendações de adubação e calagem para o Estado de São Paulo. Boletim Técnico $\mathrm{N}^{0}$ 100. 2. ed. Campinas: IAC, 1996. 285 p.

RIBEIRO, A. C.; GUIMARÃES, P. T. G.; ALVAREZ V. V. H. Recomendações para o uso de corretivos e fertilizantes em Minas Gerais: $5^{\text {a }}$ aproximação. Viçosa, MG: Comissão de Fertilidade do solo do Estado de Minas Gerais, 1999. 180 p.

ROQueJAni, M. S.; MElO, A. M. T de; SHIRAhIGE, F. H.; MELO, P. C. T de; PURQUERIO, L. F. V. Produtividade e qualidade de híbridos de tomate dos segmentos italiano e santa cruz em ambiente protegido. 2008. In: FAPESP. Anais eletrônicos... Campinas: IAC. Disponível em: <http://www.iac.sp.gov.br/ areadoinstituto/pibic/anais/2008/Artigos/RE0800020.pdf>. Acesso em: 15 jul. 2013.

SARA MEJÍA, M. T.; EDGAR, I.; ESTRADA, S.; FRANCO, P. M. Respuesta Del tomate chonto cultivar Unapal Maravilla, a diferentes concentraciones de nutrientes. Acta Agronómica, Colômbia, v. 56, n. 2, p 75-83, 2007.

SARRUGE, J. R. Soluções nutritivas. Summa Phytopathologica, Jaguariuna, v. 1, n. 3, p.231-233, 1975.

SILVA, N. M.; CARVALHO, L. H.; QUAGGIO, J. A. Ensaio de longa duração com calcário e cloreto de potássio na cultura do algodoeiro. Bragantia, Campinas, v. 54, n. 1, p.353-360, 1995.

TAIZ, L.; ZEIGER, E. Fisiologia Vegetal. 3. ed. Porto Alegre: Artmed, 2004. 719 p.

VALÉRIE, G; WIM, B; EWELINA, H; CARMONA-TORRES, C; WANG, H; VAN DE PEPPEL, A; CÓNDOR GOLEC, A; DORAIS, M; VAN MEETEREN, U; HEUVELINK, E; REMBIALKOWSKA, E; VAN BRUGGEN, A. Differences in $\mathrm{N}$ uptake and fruit quality 
between organically and conventionally grown greenhouse tomatoes. Agronomy for Sustainable Development, Paris, v. 30, n. 4, p.797-806, 2010.

VILLAS BOAS, R. L.; SOUZA, T. R. Fertirrigação: uso e manejo. In: SIMPÓSIO EM SISTEMAS AGROSILVIPASTORIS, 1, 2008, Campina Grande. Anais... Campina Grande: PPGZ/CSTR/UFCG, 2008. p. 1-14. 